Cite this: RSC Adv., 2014, 4, 20677

Received 23rd January 2014

Accepted 24th April 2014

DOI: $10.1039 / c 4 r a 00668 b$

www.rsc.org/advances

\section{Fluorescent and magnetic dual-responsive coreshell imprinting microspheres strategy for recognition and detection of phycocyanin $\dagger$}

\begin{abstract}
Zhong Zhang, ${ }^{\text {ac }}$ Jinhua Li, ${ }^{a}$ Junqing $\mathrm{Fu}^{\mathrm{ab}}$ and Lingxin Chen*a
Molecular imprinting as a versatile technology is emerging for diverse species in various fields; however protein imprinting faces several problems related to the size, structural complexity, conformational flexibility, and compatibility with solvents. Herein, by using phycocyanin as a model, with physiological significance and fluorescence characteristics, we developed a facile and highly efficient approach to obtain fluorescent and magnetic dual-responsive coreshell imprinting microspheres. Twostage miniemulsion polymerization was employed, based on surface immobilization of phycocyanin with aminolysis and aldehyde modification on superparamagnetic support particles. The dual-responsive imprinting microspheres exhibited high adsorption capacity of $10.53 \mathrm{mg} \mathrm{g}^{-1}$, excellent binding selectivity toward phycocyanin with a high imprinting factor of 2.41 , and good reproducibility with standard error within $10 \%$. Furthermore, fast simple magnetic separation and sensitive fluorescent detection in a wide $\mathrm{pH}$ range was offered for phycocyanin, showing a good linearity within $0.01-1.0 \mathrm{mg} \mathrm{L}^{-1}\left(R^{2}=0.9970\right)$ and a favorable detectability up to $1.5 \mathrm{ng} \mathrm{mL}^{-1}$. Consequently, the imprinting microspheres were successfully applied as sorbents for selective isolation of phycocyanin from protein mixtures and special imaging recognition. Taking advantages of dual-responsive polymers and surface imprinting, the developed strategy provides great application potentials for convenient, rapid targeting identification/enrichment and separation of proteins and thereby contributing to targeting drug delivery and protein research.
\end{abstract}

\section{Introduction}

Synthetic micro/nano-sized polymers capable of binding to specific biomacromolecules are of significant interest as substitutes for antibodies. ${ }^{\mathbf{1 - 4}}$ Molecularly imprinted polymers (MIPs) are well known to be one such class polymers, which is synthesized by molecular imprinting technology for creation of tailor-made binding sites with memory of the shape, size and functional groups of the template molecules. ${ }^{4,5}$ The polymers possess many promising characteristics, such as high selectivity, high stability to harsh chemical/physical conditions, low cost and easy preparation, and excellent reusability, which can overcome the shortcomings of antibodies. ${ }^{4,5}$ As a type of attractive materials, MIPs have received wide concerns in various application fields, such as purification/separation, ${ }^{6-8}$ chemo/biosensors, ${ }^{9,10}$ catalysis ${ }^{11,12}$ and drug delivery. ${ }^{13-15}$ However, attempts to

${ }^{a}$ Key Laboratory of Coastal Environmental Processes and Ecological Remediation, Yantai Institute of Coastal Zone Research, Chinese Academy of Sciences, Yantai 264003, China. E-mail: lxchen@yic.ac.cn; Fax: +86 535 2109130; Tel: +86 535 2109130

${ }^{b}$ College of Chemistry and Chemical Engineering, Qufu Normal University, Qufu 273165, China

'University of Chinese Academy of Sciences, Beijing 100049, China

$\dagger$ Electronic supplementary information (ESI) available. See DOI: $10.1039 / \mathrm{c} 4 \mathrm{ra00668b}$ extend imprinting technology and MIPs to larger templates such as proteins, have failed to show similar successes to small molecules. ${ }^{\mathbf{1 6 - 1 8}}$ This is primarily due to key inherent properties of proteins, such as large size, complex structure, flexible conformation, easy denaturation, the use of water as solvent and a large number of functional groups available for recognition, and heterogeneity in binding pocket affinity, which make it quite difficult to use imprinting protocols of small molecules for proteins. ${ }^{4,5,16-18}$ In addition, the large molecular size of proteins results in poor mass transport and permanent entrapment within traditionally dense MIP gels, while high elasticity and porosity of a looser gel makes the imprinting process less selective. ${ }^{\mathbf{1 8 - 2 0}}$ Consequently, it is still a severe challenge to find efficient and general ways to imprint proteins.

Recently, molecular imprinting on the surface of matrices has exhibited good prospects in MIPs field especially for protein imprinting. ${ }^{21-24}$ Compared to traditional MIPs, surface imprinting polymers can provide more complete removal of templates, better site accessibility, lower mass transfer resistance, and well-defined material shape. ${ }^{21-25}$ Surface imprinting is increasingly utilized, and many particles have been adopted as supports, including silica nanoparticles, ${ }^{26}$ silica gel, ${ }^{27} \mathrm{Fe}_{3} \mathrm{O}_{4}$ particles, ${ }^{28}$ polystyrene beads, ${ }^{29}$ quantum dots, ${ }^{30}$ and so on. Among them, $\mathrm{Fe}_{3} \mathrm{O}_{4}$ magnetic particles show attractive applicability, because they are non-swelling inorganic materials, 
possess high thermal resistance and high efficiency, and can be easily isolated/collected and recycled by the use of an external magnetic field. ${ }^{31,32}$

The magnetic susceptibility endowed on particles has widened the potential application scope of MIPs. ${ }^{31-34}$ Magnetic nanoparticles can be manipulated by magnetic fields, so that when functionalized (e.g., by MIPs), they can be used for the purification and separation of biomolecules and even whole cells. Besides that, the imprinted particles can also be prepared for the orientation recognition of proteins which are abundantly expressed on tumor tissues, and the superparamagnetic imprinted particles will be concentrated at the tumor site for affinity, which allows the imaging of the tumor through magnetic resonance imaging. So, excitingly, the magnetic responsive MIPs especially for proteins have increasingly boomed lately, demonstrating ideal template binding properties in aqueous media. ${ }^{33,34}$ Nevertheless, to the best of our knowledge, no fluorescent and magnetic dual-responsive MIPs have been reported for some special proteins such as phycocyanin.

Phycocyanin is a blue colored photosynthetic accessory pigment protein that emits fluorescence at about 615-640 nm, with a molecular mass of about $110 \mathrm{kDa}$ containing two subunits. ${ }^{35}$ Phycocyanin has been shown to suppress allergic inflammation reactions in different animal models. ${ }^{36}$ Besides a potential use for therapeutic purposes, phycocyanin has attracted great attention in biomedical research as a fluorescent marker. ${ }^{37}$ Moreover, phycocyanin often used as the index of cyanobacteria, occupies important environmental significance. ${ }^{38}$ Hence, phycocyanin research is becoming an emerging issue, and thereby pushing forwards productive organisms and environmental/healthy protection. Therefore, it is urgently required to develop new technologies and methods for phycocyanin identification, isolation and purification. ${ }^{39}$

Inspired by these studies, we proposed a facile and effective method to prepare phycocyanin MIPs for fluorescence and magnetic dual-response by using twostage coreshell miniemulsion polymerization based on surface imprinting of $\mathrm{Fe}_{3} \mathrm{O}_{4}$ support particles. The polymers were full characterized by Fourier transform infrared spectroscopy, element analysis, vibrating sample magnetometer, fluorescence spectroscopy/ microscopy, UV/Vis, and confocal laser scanning microscope. Molecular binding capacity and recognition specificity and fluorescent detectability were investigated in detail. Accordingly, the MIPs were successfully applied as sorbents in SDSPAGE. By combining the separation ability of $\mathrm{Fe}_{3} \mathrm{O}_{4}$ particles with the functional capability of MIPs, the dual-responsive composites proved potential applicable to target protein enrichment and fluorescence imaging analysis.

\section{Experimental}

\subsection{Reagents and materials}

Phycocyanin was kindly provided by Shandong Oriental Ocean Company (Yantai, China) and used as the template protein, and lysozyme (LZM), albumin from chicken egg (CEA), bovine serum albumin (BSA), methl methacrylate (MMA) and ethylene glycol dimethacrylate (EGDMA) were purchased from Sigma-Aldrich
(Shanghai, China). Sodium dodecyl sulfate (SDS), sodium bisulfite, ammonium persulfate (APS), and sodium bicarbonate were purchased from Shanghai Chemical Reagents Company (Shanghai, China). Sodium hydroxide, glutaraldehyde, iron(III) chloride hexahydrate, oleic acid, sodium acetate, polyethylene glycol, ethylene glycol, cetyl alcohol, and ethylene diamine were purchased from Tianjin Reagent Plant (Tianjin, China). Ammonia solution, hydrochloric acid, ethanol, $N, N$-dimethylformamide (DMF) and other affiliated chemicals were all obtained from Sinopharm Chemical Reagent Co. Ltd. (Shanghai, China). All solvents and chemicals were of analytical grade and used directly without further purification unless otherwise specified.

\subsection{Preparation of $\mathrm{Fe}_{3} \mathrm{O}_{4}$ microsphere}

$\mathrm{Fe}_{3} \mathrm{O}_{4}$ microsphere was prepared by the hydrothermal method. ${ }^{40}$ $\mathrm{FeCl}_{3} \cdot 6 \mathrm{H}_{2} \mathrm{O}(2.0 \mathrm{~g})$ and sodium acetate $(4.8 \mathrm{~g})$ were dissolved in $60 \mathrm{~mL}$ ethylene glycol to form a clear solution. Polyethylene glycol (2.6 g) was added with mechanical mixing vigorously until dissolved. The mixture was stirred for $30 \mathrm{~min}$ at $30{ }^{\circ} \mathrm{C}$. Then the resulting clear yellowish green solution was transferred to a telfon hot water kettle. The kettle was heated to and maintained at $200{ }^{\circ} \mathrm{C}$ for $10 \mathrm{~h}$, and then it was cooled to room temperature. The collected magnetic products were purified in turn with ultrapure water produced by a Milli-Q Ultrapure Water System (Millipore, Bedford, MA, USA) and anhydrous ethanol washing 6 times, and dispersed in water for subsequent use.

\subsection{Synthesis of superparamagnetic support particles}

Superparamagnetic support particles were synthesized by miniemulsion polymerization following the process described..$^{41} \mathrm{Fe}_{3} \mathrm{O}_{4}(0.5 \mathrm{~g})$ prepared above was dispersed in oleic acid $(1.0 \mathrm{~mL})$ to obtain a black viscous gel, followed by the addition of MMA $(0.64 \mathrm{~mL})$ and EGDMA $(4.52 \mathrm{~mL})$ and then mixed thoroughly with ultrasonic. The resulting mixture was added dropwise into $25 \mathrm{~mL}$ solution containing 0.01 M SDS and $0.03 \mathrm{M}$ cetyl alcohol, which was mechanically stirred vigorously to form a miniemulsion. The miniemulsion was then added dropwise into $300 \mathrm{~mL}$ SDS solution $(0.05 \%, \mathrm{w} / \mathrm{v})$. And then the mixture was transferred to a $500 \mathrm{~mL}$ round-bottom flask with APS $(0.25 \mathrm{~g})$ added. The polymerization was undertaken with mechanically stirring in nitrogen atmosphere at $70{ }^{\circ} \mathrm{C}$ for $24 \mathrm{~h}$. Afterward, the polymers were washed with ultrapure water/ methanol repeatedly with the aid of an external magnetic field.

\subsection{Aminolysis}

Particles ( $1 \mathrm{~g}$ ) prepared above was washed twice with DMF with the aid of an external magnetic field. Then the particles were redispersed in $20 \mathrm{~mL}$ DMF with ultrasonic, followed by the addition of $20 \mathrm{~mL}$ ethanediamine to the mixture and vigorously mechanically stirring for $12 \mathrm{~h}$ under reflux at $110{ }^{\circ} \mathrm{C}$. The amine-group particles were washed with ultrapure water/ethanol repeatedly.

\subsection{Modification with aldehyde group}

The amine-group particles ( $1 \mathrm{~g}$ ) prepared above was washed twice with $\mathrm{pH} 5$ phosphate buffer solution (PBS). Then the 
particles were re-dispersed in $10 \mathrm{~mL}$ PBS with ultrasonic. Then $0.5 \mathrm{~mL}$ glutaraldehyde was added and purged with nitrogen gas for $10 \mathrm{~min}$ to displace oxygen. The polymerization was undertaken with mechanically stirring for $12 \mathrm{~h}$ at room temperature. The aldehyde group particles were then washed three times with ultrapure water.

\subsection{Preparation of magnetic imprinted polymers}

The attained particles $(1.0 \mathrm{~g})$ above were then added into $10 \mathrm{~mL}$ phycocyanin solution $\left(5 \mathrm{mg} \mathrm{mL} \mathrm{m}^{-1}\right)$. The mixture was mechanically stirred for $3 \mathrm{~h}$ at $4{ }^{\circ} \mathrm{C}$ in an ice bath to promote protein coupling. The phycocyanin-immobilized particles were collected with external magnetic field, and then were washed two times with ultrapure water. Magnetic MIPs were synthesized by miniemulsion polymerization following the process "2.3. Synthesis of superparamagnetic support particles" described above. The different point was the initiator used in the reaction. Sodium bisulfite $(0.25 \mathrm{~g})$ followed by APS $(0.25 \mathrm{~g})$ was used in the mixture to initiate the polymerization at $40{ }^{\circ} \mathrm{C}$ for $24 \mathrm{~h}$. That is because APS only can affect high-grade structure of protein leading to protein denaturation at high temperature. Then the magnetic imprinted polymers were rinsed using ultrapure water and ethanol three times to wash off residues with the aid of an external magnetic field. Afterward, the immobilized template phycocyanin molecules in the magnetic imprinted polymers were removed by sodium hydrate solution hydrolysis. The hydrolysis mixture was stirred under nitrogen atmosphere for 6 $\mathrm{h}$ at $35^{\circ} \mathrm{C}$. These aldehyde group modified imprinted polymers were washed three times with ultrapure water and dried in a vacuum oven at $40{ }^{\circ} \mathrm{C}$ to attain the products. For simplicity, CMIP was used to indicate the products.

In order to prove the special role of aldehyde group in the combination of the protein, amino-group modified imprinted polymers (N-MIP, for simplicity) were prepared for comparison, directly from amidogen group without aldehyde group modified in the same way. As a control, non-imprinted polymers modified with aldehyde group (C-NIP, for simplicity) and non-imprinted amino-group modified imprinted polymers (N-NIP, for simplicity) were also prepared by using the same procedures and conditions, only without the addition of template phycocyanin protein.

\subsection{Characterization of the magnetic and fluorescent dual- responsive MIPs}

FT-IR spectrometer (Nicolet iS10, Thermo scientific) and element analyzer (Vario Micro cube, Elementar) were used to investigate the preparation process. Size distribution was determined by the laser particle analyzer (MASTERSIZE2000, Malvern Instruments, UK). Fluorescence spectra were conducted on a spectrofluometer (Fluoromax-4, HORIBA) for the determination of phycocyanin. Excitation light was set at $590 \mathrm{~nm}$ and the emission spectra were recorded from 605 to $700 \mathrm{~nm}$, and the silt widths of excitation and emission were 5 and $5 \mathrm{~nm}$, respectively. The magnetic property was analyzed by using a vibrating sample magnetometer (VSM). A fluorescence microscope (BX61, Olympus) was employed to observe the fluorescence microscopy image of sample on a glass slide equipped with a CCD camera, and further observations were conducted to fluorescence qualitative analysis by using confocal laser scanning microscopy (CLSM) (FV1000, Olympus). UV/Vis absorption spectra of proteins were measured on a spectrophotometer (NanoDrop 2000/2000C, Thermo Scientific) at the wavelength of $280 \mathrm{~nm}$ to test the binding properties of polymers.

\subsection{Estimation of adsorption capacity and recognition properties of the dual-responsive MIPs}

To evaluate the binding capacity of the magnetic MIPs obtained, static adsorption tests and dynamic adsorption tests for phycocyanin were carried out in aqueous solutions. The procedures were carried out as follows: $20 \mathrm{mg}$ polymer particles were put into a $5 \mathrm{~mL}$ flask containing $2.0 \mathrm{~mL}$ of phycocyanin solutions of various concentrations $\left(0.1-0.5 \mathrm{mg} \mathrm{mL}^{-1}\right)$. After 12 $\mathrm{h}$ shaking at room temperature, the supernatant solutions were collected with the aid of an external magnetic field, the concentrations of which were attained by fluorescent determination. The binding amount $(Q)$ of phycocyanin was calculated by subtracting the free concentrations from the initial concentrations. The maximum binding capacity $\left(Q_{\max }\right)$ and dissociation constant $\left(K_{\mathrm{d}}\right)$ were estimated by processing with the Scatchard equation as follows:

$$
Q / C_{\mathrm{e}}=\left(Q_{\max }-Q\right) / K_{\mathrm{d}}
$$

where, $Q$ is the amount of phycocyanin adsorbed onto the polymer at equilibrium, $C_{\mathrm{e}}$ is the free phycocyanin concentration at equilibrium, $K_{\mathrm{d}}$ is the dissociation constant and $Q_{\max }$ is the apparent maximum amount that can be bound. The $K_{\mathrm{d}}$ and $Q_{\max }$ can be obtained from the slope and intercept of the linear curve plotted according to $Q / C_{\mathrm{e}} v s$. $Q$, respectively.

Meanwhile, dynamic adsorption test was carried out by monitoring the temporal amount of phycocyanin in the solutions.

Selectivity experiments were performed by using LZM, CEA, and BSA as competitive proteins with initial concentrations of $0.5 \mathrm{mg} \mathrm{mL}{ }^{-1}$. The concentration of competitive proteins in the supernatant was measured by UV/Vis spectrometer at the wavelength of $280 \mathrm{~nm}$. All the tests were performed in triplicates. The average data from triplicate independent results were used for the following discussion.

In addition, the recognition ability of the MIPs was evaluated by using imprinting factor $(\alpha)$, which is defined as the following formula:

$$
\alpha=Q_{\mathrm{MIP}} / Q_{\mathrm{NIP}}
$$

where, $Q_{\mathrm{MIP}}$ and $Q_{\mathrm{NIP}}$ are the adsorption amounts of template or analogues on MIP and NIP at equilibrium, respectively.

\subsection{SDS-PAGE analysis of the dual-responsive MIPs}

Twenty milligram $(20 \mathrm{mg})$ of C-MIP or C-NIP was immerged in 2 $\mathrm{mL}$ of the spiked protein aqueous solutions (containing the mixture of phycocyanin, LZM, CEA and BSA individual at $1 \mathrm{mg}$ $\mathrm{mL}^{-1}$ ). After incubation for $12 \mathrm{~h}$ at room temperature under 
gently shaking, the supernatant solutions were collected with the aid of an external magnetic field and analyzed by sodium dodecyl sulfate polyacrylamide gel electrophoresis (SDS-PAGE).

\subsection{Cell experiments of the dual-responsive MIPs}

Cell experiments were carried out for C-MIP and C-NIP as described ${ }^{42}$ with necessary modifications in the followings. Hela cells were grown as a monolayer in a humidified incubator at 37 ${ }^{\circ} \mathrm{C}$ in air- $\mathrm{CO}_{2}$ (95:5) in RPMI-1640 medium that was supplemented with $10 \%$ fetal bovine serum. For all the experiments, the cells were harvested by using trypsin and were re-suspended in fresh medium before plating. And then, Hela cells were seeded onto glass cover slips in a 12 -well plate in RPMI-1640 medium that contained $10 \%$ fetal bovine serum for $24 \mathrm{~h}$ at $37^{\circ} \mathrm{C}$ in a humidified atmosphere with $5 \% \mathrm{CO}_{2}$ to allow the cells to attach. After incubation for $24 \mathrm{~h}, 60 \mu \mathrm{L}$ phycocyanin aqueous solution $\left(10 \mathrm{mg} \mathrm{mL}^{-1}\right)$ was added into the plate, and then C-MIP or C-NIP was put into the plate and incubated for $2 \mathrm{~h}$. The cell monolayer on the cover-slip was repeatedly washed with PBS $(\mathrm{pH}=7.0)$ to remove the dead cells and then sealed with a glass microscope slide. Observations were performed by CLSM at the excitation wavelength of $559 \mathrm{~nm}$.

\section{Results and discussion}

\subsection{Materials preparation and characterization}

Surface imprinting is one of the most appealing ways to prepare MIPs with cavities on the surface or close to the surface, which can solve these problems, such as slow binding kinetics, and incomplete removal of templates. Magnetic adsorbents make separation process simple and fast, because they can be easily collected by an external magnetic field without additional centrifugation or filtration. Based on the above technologies, a new procedure for preparing phycocyanin surface-imprinted polymers with magnetic susceptibilities was developed by using modified miniemulsion polymerization method, as illustrated in Fig. 1A. The synthesized magnetic support particles were hydrophobic by coating with oleic acid, which allowed them to be dispersed in the oil-phase hydrophobic mixture of MMA and EGDMA of the miniemulsion. Ethanediamine was introduced onto the surface of the polymeric support particles, and glutaraldehyde was chosen as the bridging agent as it possesses two terminal aldehyde groups. The free surface aldehyde reacted with the free amine groups in the imprinted molecules phycocyanin. An external imprinted polymeric shell was prepared on the surface of phycocyanin immobilized support beads during the second-stage miniemulsion polymerization with MMA and EGDMA as the functional monomer and crosslinker, respectively. It is able to provide ester and methoxy groups for subsequent surface functionalization. Being a weak electron donor, the esters group is susceptible to nucleophilic attack during the aminolysis substitution reaction. The addition of EGDMA as a cross-linker maintained the stability of the imprinting sites while making the product polymeric beads easier to be handled and processed. Subsequently, the phycocyanin molecules were hydrolyzed to remove from the particles,
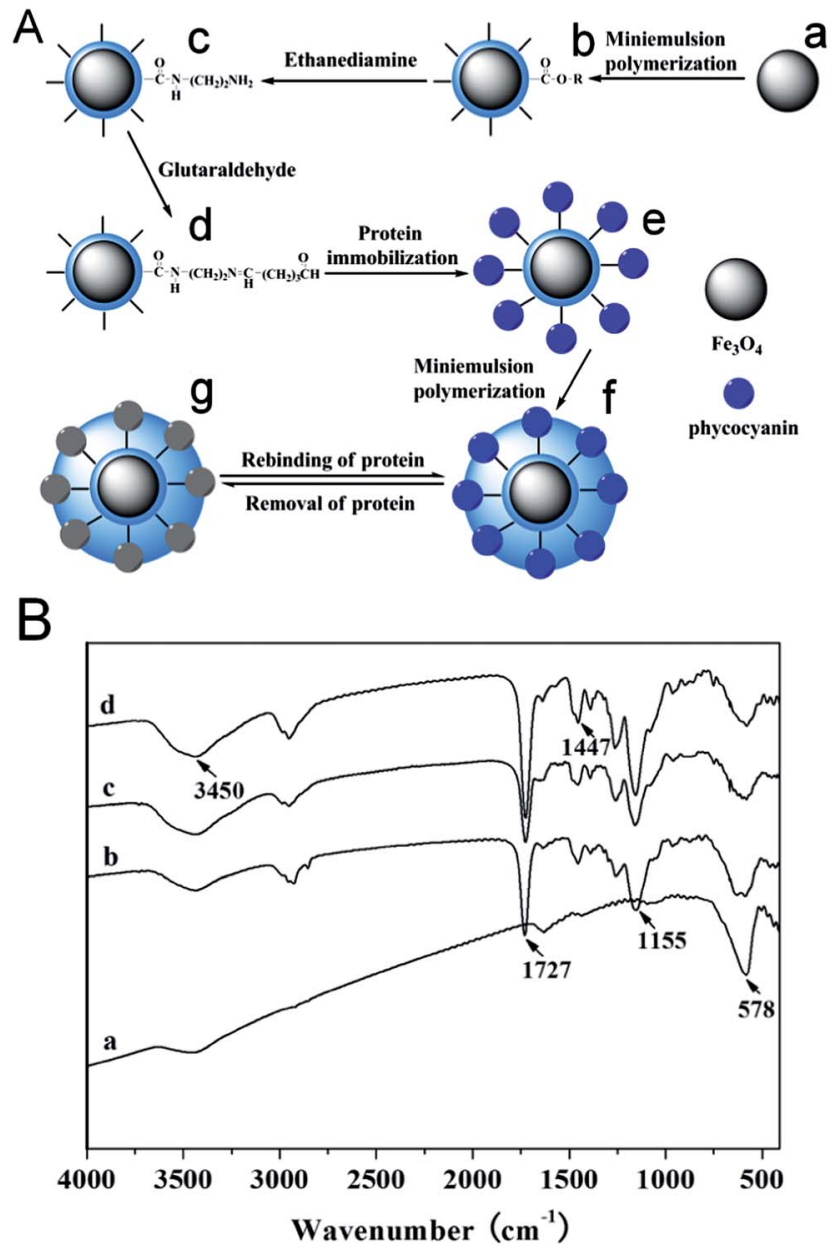

Fig. 1 (A) Schematic illustrations for the preparation process of C-MIP. (a) $\mathrm{Fe}_{3} \mathrm{O}_{4}$ microsphere, (b) magnetic support particles, (c) amine group grafted particles, (d) aldehyde group grafted particles, (e) phycocyaninimmobilized particles, (f) aldehyde group modified imprinted polymers without phycocyanin removal, and $(\mathrm{g})$ aldehyde group modified imprinted polymers. (B) FT-IR spectra of: (a) $\mathrm{Fe}_{3} \mathrm{O}_{4}$ microsphere, (b) magnetic support particles, (c) amine group grafted particles, and (d) aldehyde group modified imprinted polymers.

leaving behind binding sites with memory of the shape, size and functional groups of the phycocyanin on the particle's outer shell. Resultantly, phycocyanin coreshell MIPs were prepared.

To investigate the grafting of new activated groups on $\mathrm{Fe}_{3} \mathrm{O}_{4}$, the attained particles of each modification step were characterized by FT-IR spectra and elemental analysis. The FT-IR spectra of $\mathrm{Fe}_{3} \mathrm{O}_{4}$ particles, magnetic support particles, aminegroup particles and aldehyde group modified imprinted polymers (C-MIP) were measured, as shown in Fig. 1B. The observed peak around $580 \mathrm{~cm}^{-1}$ could be attributed to $\mathrm{Fe}-\mathrm{O}$ stretching vibration, indicating the occurrence of $\mathrm{Fe}_{3} \mathrm{O}_{4}$ matrices in the four materials. After a series of embedding and modification, the characteristic absorption peak for $\mathrm{Fe}_{3} \mathrm{O}_{4}$ became weak significantly, while other characteristic peaks appear for the CMIP. As observed in Fig. 1B(d), the wide and strong absorption band around $1727 \mathrm{~cm}^{-1}$ could be ascribed to stretching vibrations of $\mathrm{C}=\mathrm{O}$, and the absorption peaks at $3450 \mathrm{~cm}^{-1}$ belonged to the $\mathrm{N}-\mathrm{H}$ stretching vibration. The peaks around $1447 \mathrm{~cm}^{-1}$ 
Table 1 Surface atomic composition ratios of the particles from elemental analysis (\%)

\begin{tabular}{lllll}
\hline Stage & $\mathrm{N}$ & $\mathrm{C}$ & $\mathrm{H}$ & Remains \\
\hline Support particles & 0.043 & 43.551 & 6.067 & 50.339 \\
$\mathrm{NH}_{2}$ - particles & 1.224 & 40.872 & 6.144 & 51.760 \\
$\mathrm{CHO}$ - particles & 0.759 & 42.503 & 6.254 & 50.484 \\
Protein & 2.814 & 41.277 & 5.927 & 49.982 \\
immobilization & & & & \\
C-MIP & 0.378 & 42.698 & 6.124 & 50.800
\end{tabular}

suggested the $\mathrm{C}-\mathrm{N}$ had been successfully grafted onto the surface of the magnetic support particles after modification. Moreover, the peaks at about 1262 and $1155 \mathrm{~cm}^{-1}$ could be ascribed to the stretching vibration absorption of saturated $\mathrm{C}-\mathrm{O}$ band in ester groups, indicating that ester groups in EGDMA had been successfully grafted onto the surface of the $\mathrm{Fe}_{3} \mathrm{O}_{4}$. These results confirmed the phycocyanin C-MIP was successfully prepared by twostage miniemulsion polymerization, based on the surface immobilization of template phycocyanin molecules with aminolysis and aldehyde modification on the superparamagnetic support particles. Moreover, the size distributions of C-MIP was obtained by laser particle analyzer. As evidenced in Fig. S1, $\dagger$ the intensity/volume contribution versus diameters of microparticles displayed a good size distribution and a dominant distribution peak was around $10.5 \mu \mathrm{m}$.

Elemental analysis results were recorded in Table 1. As observed, the nitrogen ratio obtained for relevant particles that contain amidogen groups was well consistent with the above mentioned chemical modifications. The increase in the nitrogen atomic composition from 0.043 to $1.224 \%$ after the first aminolysis explained that amine groups were successfully introduced onto the surfaces of $\mathrm{Fe}_{3} \mathrm{O}_{4}$. After the aldehyde functionalization, the decrease in the nitrogen composition to $0.759 \%$ accompanying with the increase of carbon composition from 40.872 to $42.503 \%$ might be indicative of the relative increase in carbon and oxygen content from glutaraldehyde. The successful immobilization of phycocyanin could be seen by the significant increase in the nitrogen composition from 0.759 to $2.814 \%$. This increase was owing to the abundant peptide bonds from the protein molecules. The phycocyanin was hydrolyzed to remove from the particles along with the nitrogen composition down to $0.378 \%$. These experimental data were able to provide direct evidence on the chemical modification of particles.

\subsection{Magnetic properties of the MIPs}

In the preparation of the C-MIP for potential magnetic separation, it is important that the material should possess sufficient magnetic property for practical application. In order to characterize the magnetism property, VSM analysis was employed. The magnetization curves from the VSM analysis were shown in Fig. 2. The saturation magnetization values of C-NIP and C-MIP were about 12.26 and $10.77 \mathrm{emu} \mathrm{g}^{-1}$, respectively. The existence of protein cavities on the surface of C-MIP could be most likely

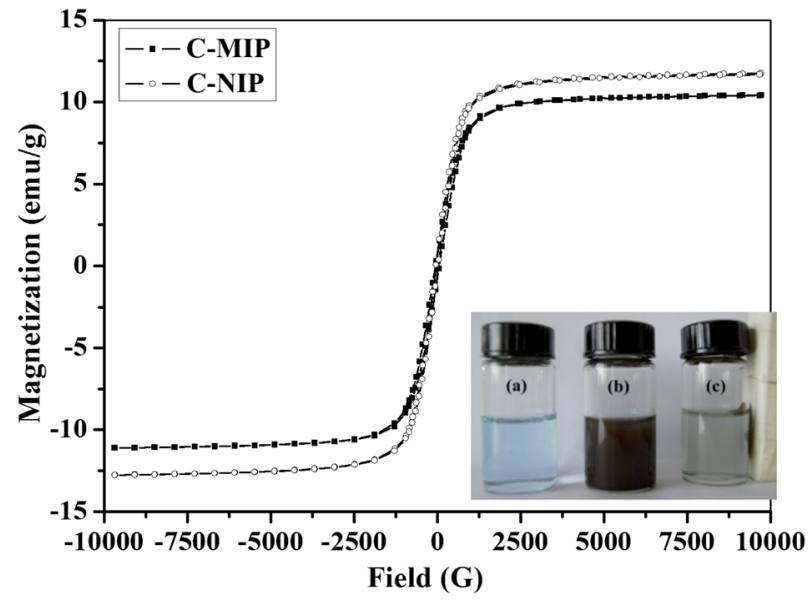

Fig. 2 VSM magnetization curves of C-MIP and C-NIP, and the insert shows the separation and redispersion process of (a) phycocyanin solution, (b) phycocyanin solution with C-MIP, (c) phycocyanin solution with C-MIP and presence of an external magnetic field.

to contribute to the slight decrease of the saturation magnetization. Interestingly, the curve suggested that the materials could respond magnetically to an external magnetic field effectively and could well disperse when removing the field. As proved in the inset of Fig. 2, in the absence of an external magnetic field, a homogeneous dispersion was demonstrated; when an external magnetic field was applied, the black particles were attracted to the vial wall in a short time (about $3 \mathrm{~min}$ ). Thus, the saturation magnetization value achieved for the $\mathrm{C}$ MIP was sufficiently high for magnetic separation in practical applications.

\subsection{Binding properties of the MIPs for phycocyanin}

The adsorption isotherm experiments for the prepared C-MIP, N-MIP and their corresponding NIPs were carried out at different initial concentrations of phycocyanin, ranging from 0 $0.5 \mathrm{mg} \mathrm{mL} \mathrm{m}^{-1}$. As shown in Fig. S2a, $\dagger$ the adsorbed amount of phycocyanin per unit mass of the polymer increased along with the increasing initial concentrations, while the binding amounts of both C-MIP and N-MIP for phycocyanin were much higher than that of their corresponding NIPs. The results could be explained that MIPs with specific recognition cavities showed high binding affinity for template. On the contrary, there were no specific recognition sites on the NIPs, so the binding capacity of NIPs was the lowest. Moreover, the binding amounts of C-MIP were higher than that of N-MIP, and this could be attributed to the combined effect of aldehyde and amine groups, which involved nucleophilic addition that allowed the formation of imine bonds.

The Scatchard equation could be employed to estimate the binding capacity of the polymers. Accordingly, the equilibrium dissociation constant $K_{\mathrm{d}}$ and the maximum binding capacity $Q_{\max }$ of the two MIPs were calculated. That is, $Q_{\max }$ values for CMIP and N-MIP were 10.53 and $9.18 \mathrm{mg} \mathrm{g}^{-1}$, respectively, and $K_{\mathrm{d}}$ values were 0.109 and $0.113 \mathrm{mg} \mathrm{mL}^{-1}$, respectively. Meanwhile, the $Q_{\max }$ value for C-NIP was $4.37 \mathrm{mg} \mathrm{g}^{-1}$, and thereby the $Q_{\max }$ 
ratio (10.53 to 4.37) for C-MIP was obtained of 2.41. On the other hand, in this work the imprinting factor $(\alpha)$, which was defined as the ratio of the capacities of C-MIP and C-NIP at equilibrium state, i.e., 8.36 and $3.47 \mathrm{mg} \mathrm{g}^{-1}$, respectively, experimentally determined, was also 2.41. So, the theoretical imprinting effect results were well in consistent with the experimental results. So, it suggested the C-MIP possessed unique template molecule adsorption properties. As shown in Fig. S3, $\uparrow$ a straight line with a good linearity was given, suggesting there was mainly one kind of binding sites for the C-MIP. And, the rebinding was primarily related to the specific recognition sites with high binding affinity for phycocyanin..$^{\mathbf{4 1}}$ In contrast, the interaction of C-NIP with phycocyanin was mainly from nonspecific adsorption such as van der Waals. Moreover, it is well known that the Freundlich and Langmuir isotherm models are generally used to account for heterogeneous binding sites in MIPs. ${ }^{43}$ Related model parameters could be calculated according to their corresponding equations, ${ }^{43-45}$ as listed in Table $\mathrm{S} 1 . \dagger$ It can be found that the Langmuir isotherm model yielded a better fit than that by the Freundlich model for the two MIPs with higher correlation coefficients. In addition, the values of dimensionless separation factor were both between 0 and 1.0, further confirming that the Langmuir isotherm model was suitable to the MIPs adsorption for phycocyanin. ${ }^{43}$

The adsorption kinetics property is one of the most important considerations for the practical application of MIPs. It can be seen from Fig. $\mathrm{S} 2 \mathrm{~b} \dagger$ that all the three polymers had relative high initial adsorption rates that decreased slowly over time to finally achieve equilibrium. It was observed that MIPs exhibited much higher binding capacity and faster mass transfer than NIPs. This suggested the template adsorption for the C-NIP could be nonspecific, and longer time would probably be required for the template molecules to orient themselves to the surface of C-NIP. For MIPs, there was no significant variation between the rebinding kinetics for the C-MIP and N-MIP. The adsorption kinetics was favorable, reaching equilibrium $(>95 \%$ completion) in $40 \mathrm{~min}$. This phenomenon could be explained as follows: by combining surface imprinting with micro-sized polymers, most recognition sites were situated on the surface of the C-MIP, which facilitated the mass transfer. Therefore, the formation of surface-imprinted micro/nanoparticles is highly desirable to improve the binding capacity, and to enable the equilibrium to be achieved within a shorter period of time.

To evaluate the recognition specificity of C-MIP towards template phycocyanin, other three proteins with a wide range of isoelectric point (pI) and molecular weight $\left(M_{\mathrm{w}}\right)$, including LZM, CEA and BSA, were chosen as the controls. Fig. S2c $\dagger$ presents the rebinding capacities of the C-MIP and C-NIP for these proteins. It can be seen that the C-MIP had excellent binding selectivity for phycocyanin with respect to C-NIP, and its corresponding imprinting factor $(\alpha)$ reached 2.41, much higher than those structural analogs. The results indicated that the imprinting process created a microenvironment with the complementarity in shape, size and functional groups that can exactly match the template molecule. On the contrary, for CNIP, there was no tailor-made recognition sites formed, so CNIP bound the structural analogs only by non-specific adsorption. As a result, C-NIP adsorbed the template molecules much less than the C-MIP, and there was no significant difference in binding capacity for phycocyanin and its competitive proteins. Thus, the C-MIP was demonstrated highly specific to phycocyanin recognition and adsorption.

Reusability is also an important index for the novel C-MIP, which is very likely to be a key factor in improving the economical efficiency. Adsorption-desorption cycle was repeated five times by using the same imprinted polymers. When $1.0 \mathrm{~mol} \mathrm{~L}^{-1} \mathrm{NaOH}$ solution was used as desorption medium for $3 \mathrm{~h}$, desorption ratio reached nearly $100 \%$ after treatment. It was very simple and efficient since the hydrolysis mixture could be removed with the aid of an external magnetic field. As shown in Fig. S2d, $\uparrow$ the C-MIP displayed high binding capacities and removal efficiency, with only a slight decrease within $10 \%$. The reduction was probably caused by the loss of CMIP from the rinsing process. Nevertheless, the imprinting factor is still kept constant with a high value. The results indicated that the prepared C-MIP had excellent reuse/regeneration ability, which is favorable to real-world applications.

\subsection{Fluorescent quantitation of the C-MIP for phycocyanin}

Phycocyanin has good fluorescence properties in aqueous solution which possesses a lot of advantages used as a fluorescent marker, such as a wider spectral range with high absorption coefficient, and very high fluorescence quantum yield in a wide $\mathrm{pH}$ range. The fluorescence intensities of phycocyanin at the $\mathrm{pH}$ range of 5.6-8.0 were determined, as shown in Fig. 3a. The phycocyanin solution had a maximum fluorescence emission at $640 \mathrm{~nm}$ at $\mathrm{pH} 6.4$ when excited at 590 $\mathrm{nm}$, so the following determinations of the samples were conducted in the buffer solution with $\mathrm{pH}$ 6.4. Near neutral environmental was proved potentially applicable, which is most desirable to protein and physiological researches. It can be seen from Fig. $3 \mathrm{~b}$ that the fluorescence intensity increased with the increasing concentration of phycocyanin. Furthermore, through C-MIP treatment, a calibration curve for phycocyanin was obtained by using fluorescence intensity measured at 640 $\mathrm{nm}$, with an excellent linearity within $0.01-1.0 \mathrm{mg} \mathrm{L}^{-1}\left(R^{2}=\right.$ 0.9970), as well as a favorable limit of detection (LOD) was achieved of $1.5 \mathrm{ng} \mathrm{mL}^{-1}$ based on the signals as 3-fold the baseline noise. So, phycocyanin could be fluorescently quantified by the C-MIP. The fluorescence property of C-MIP was also confirmed using fluorescence microscopy. As can be seen in Fig. S4, $\uparrow$ C-MIP exhibited strong fluorescence in the presence of phycocyanin. However, no fluorescence was found without phycocyanin, and the C-MIP particles could be found in the corresponding bright-field image.

\subsection{Separation and imaging of phycocyanin via the dual- responsive C-MIP}

To evaluate the practicality of the developed materials, accordingly, the prepared magnetic and fluorescent dual-responsive CMIP with high binding capacity and rapid kinetics and recognition specificity, were further applied for the separation of phycocyanin from other protein mixture. Fig. 4 presents the 

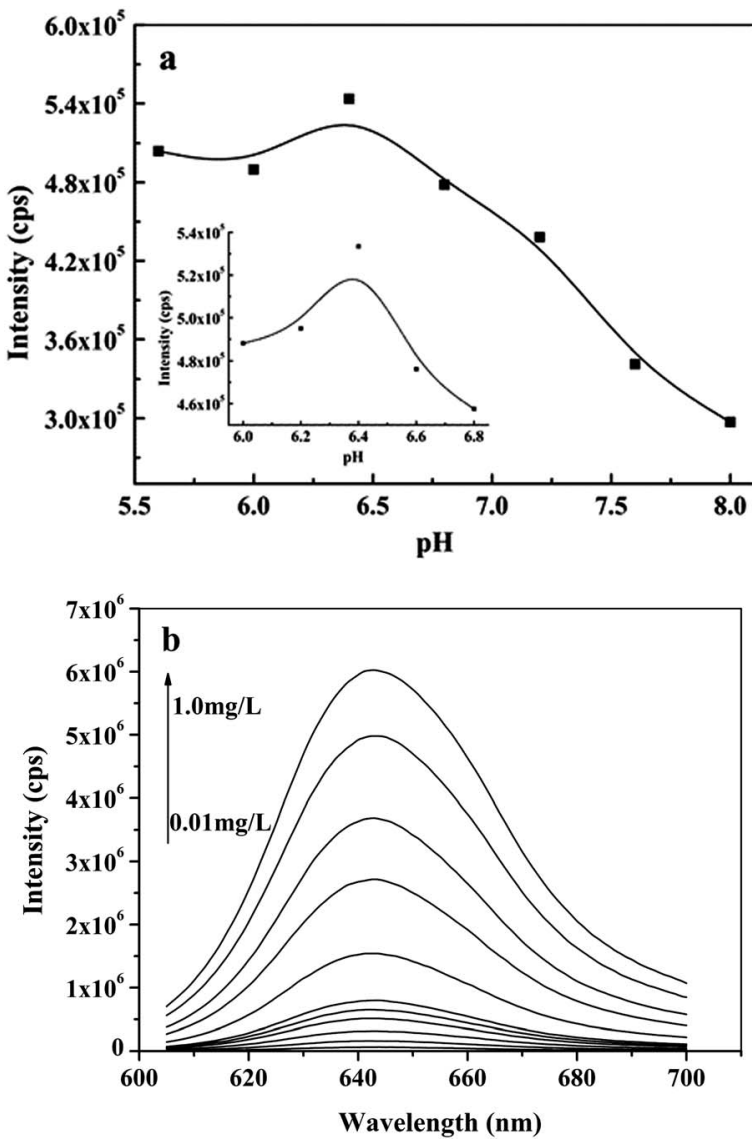

Fig. 3 (a) Fluorescence intensity of phycocyanin solution with different $\mathrm{pH}$ values, inset of (a) with $\mathrm{pH}$ 6.0-6.8; (b) fluorescence emission spectra with increasing phycocyanin concentrations in aqueous solutions after C-MIP treatment. Experimental conditions: the excited light was $590 \mathrm{~nm}$, and the silt widths of excitation and emission were 5 and $5 \mathrm{~nm}$, respectively.

SDS-PAGE results. As seen from the figure, the separation of protein mixture without treatment revealed four main bands in the lane (2), indicating the presence of the mixture of LZM, phycocyanin, CEA and BSA according to molecular weight. After treatment with C-MIP, the supernatant was detected. It was seen from lane (3) that the band of phycocyanin disappeared obviously and the remainders had little change, suggesting most of phycocyanin was specifically captured by C-MIP. Different from lane (3), there were still four bands observed in lane (4) after treatment with C-NIP. The result further validated that the NIPs had no selectivity towards phycocyanin. On the other hand, through gray level determination by software BandScan 5.0, we could attain the follow information: in lane (2), the present amount of phycocyanin was estimated $24.9 \%$ of the total protein; after treatment with C-MIP, in lane (3), phycocyanin decreased to $9.7 \%$, which could be roughly quantified that $38.9 \%$ (the ratio of $9.7 \%$ to $24.9 \%$ ) phycocyanin was remained while $61.1 \%$ phycocyanin was adsorbed onto C-MIP. It was well in agreement with the adsorption data from fluorescence analysis (from 1 to $0.47 \mathrm{mg} \mathrm{mL} \mathrm{m}^{-1}$ ). Therefore, it is practically feasible using the C-MIP for selective isolation and enrichment of phycocyanin from protein mixtures via magnetic separation.
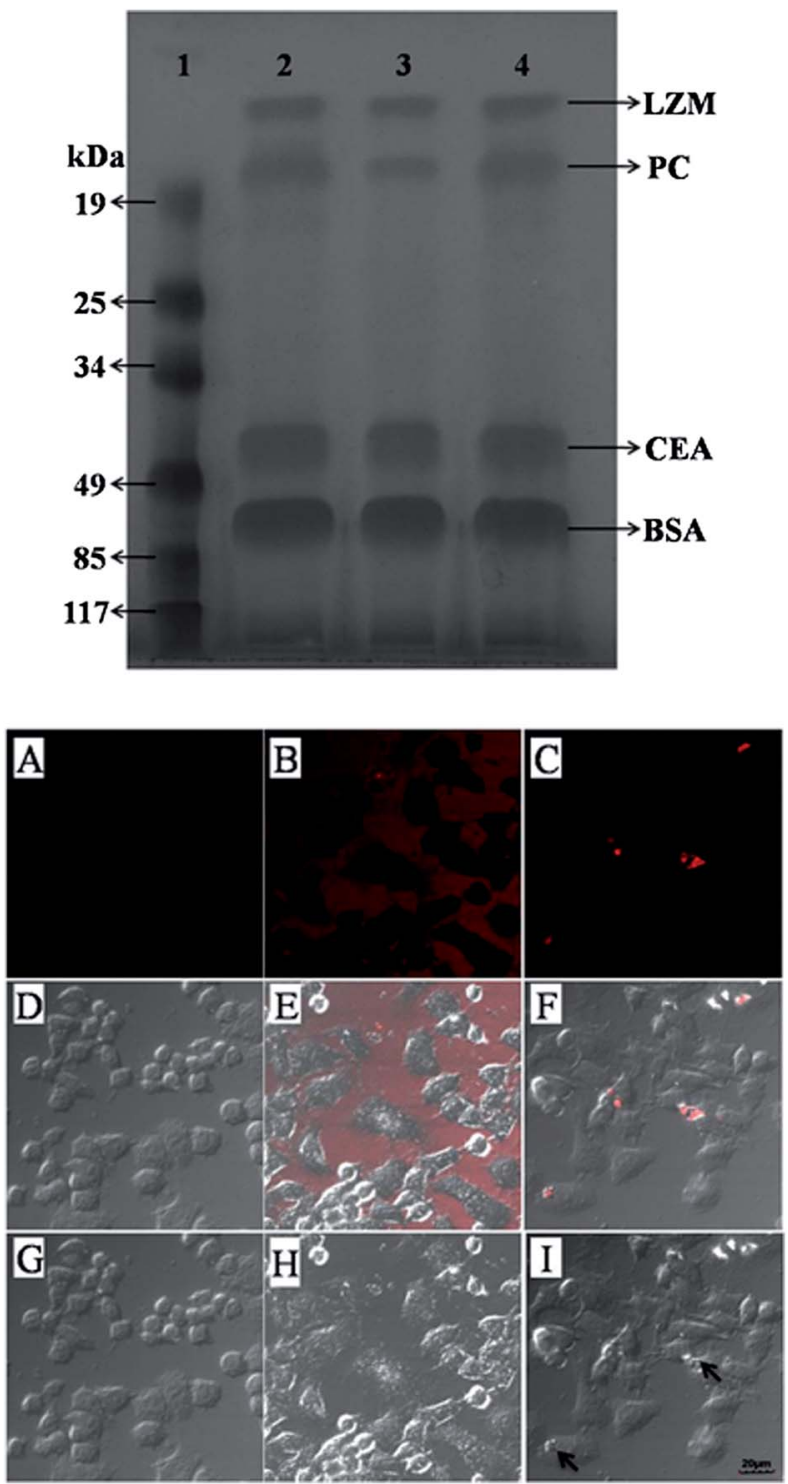

Fig. 4 (Up) SDS-PAGE analysis of spiked protein mixture treated with C-MIP and C-NIP: lane (1), marker protein; lane (2), spiked protein mixture with phycocyanin (PC), LZM, CEA and BSA; lane (3), the supernatant after treatment with C-MIP; and lane (4), the supernatant after treatment with C-NIP. Experimental conditions: loading amount of protein mixtures: $10 \mu \mathrm{L}$. (Low) confocal microscopy images of $\mathrm{C}$ MIP adsorbing phycocyanin in living Hela cell environment: (A) fluorescence image of Hela cells at the excitation wavelength of $559 \mathrm{~nm}$; (B) fluorescence image of Hela cells incubated with phycocyanin for 2 $\mathrm{h}$ at $37^{\circ} \mathrm{C}$; and (C) fluorescence image of Hela cells incubated with phycocyanin for $2 \mathrm{~h}$ at $37^{\circ} \mathrm{C}$, with the addition of C-MIP for $30 \mathrm{~min}$. (G) Bright-field image of $(A),(H)$ bright-field image of $(B)$, and (I) brightfield image of (C). (D) Overlay image of (A) and (G), (E) overlay image of (B) and (H), and (F) overlay image of (C) and (I). All scale bars are $20 \mu \mathrm{m}$.

Furthermore, the magnetic and fluorescent dual-responsive C-MIP was applied to living cellular environment for imaging examination. Before cell imaging, protein cytotoxicity was assessed. The assay in Hela cells with phycocyanin concentrations of $10 \mathrm{mg} \mathrm{mL}^{-1}$ of $60 \mu \mathrm{L}$ incubated for $24 \mathrm{~h}$ at $37^{\circ} \mathrm{C}$, clearly demonstrated that the phycocyanin was of low toxicity to cultured cell lines. We next assessed the ability of the C-MIP to 
selective recognition of phycocyanin in living cellular environment. Hela cells were incubated for $24 \mathrm{~h}$, and $60 \mu \mathrm{L}$ phycocyanin aqueous solution $\left(10 \mathrm{mg} \mathrm{mL}^{-1}\right)$ was added into the plate, and then C-MIP was put into the plate and incubated for $30 \mathrm{~min}$. The cell monolayer on the cover-slip was repeatedly washed with PBS ( $\mathrm{pH}=7.0$ ) to remove the dead cells and then sealed with a glass microscope slide. Observations were performed by CLSM at the excitation wavelength of $559 \mathrm{~nm}$, where the phycocyanin emitted red fluorescence.

Fig. 4 also shows a typical set of cell imaging micrographs. As seen, Hela cells could not be seen without phycocyanin (Fig. 4A) since they cannot emit fluorescence, and they could be found in corresponding bright-field (Fig. 4G). Upon addition of phycocyanin, an obvious intracellular fluorescence was observed in the phycocyanin treated cells (Fig. 4B and E) with respect to control samples in which the fluorescence ratiometric signal was relatively weak. And then C-MIP was put into the plate and incubated for $2 \mathrm{~h}$. The intracellular fluorescence became weak in the same condition, while interestingly, a gradual increase of bright spot appeared after incubating the cells with C-MIP for $30 \mathrm{~min}$ (Fig. 4C). The C-MIP sample could be found in the corresponding bright-field image (Fig. 4I). All these results indicated that phycocyanin was a good candidate as a fluorescent marker in living cells, and the C-MIP could realize the selective recognition and adsorption of phycocyanin in living cellular environment.

\section{Conclusions}

In summary, a new type of fluorescent and magnetic dualresponsive protein imprinting microspheres, was smartly prepared by combining the surface imprinting technique with coreshell miniemulsion polymerization, and they were successfully applied to selective recognition and sensitive detection of phycocyanin. The obtained microspheres displayed the fluorescent properties of phycocyanin and showed a response to an external magnetic field. Excellent features were demonstrated, such as remarkable fluorescent properties towards the template, high adsorption capacity, fast rebinding kinetics and high selectivity, as well as simple and rapid separation and good reproducibility. The idea of dual-responsive imprinting microspheres presented here makes possible fluorescent detection and fluorescent imaging of proteins, and the magnetic property allows for magnetic separation, showing convenient, cost-effective and environmentally friendly, which provides a new method for protein analysis and abatement. This strategy might well be an ideal stepping stone to realize the identification and removal of proteins abundantly expressed on tumor tissues with superparamagnetic imprinting particles, as well as the effective enrichment and quantification of low abundance proteins. Therefore, more related efforts should be made to push forwards the development of targeted proteomics and magnetic resonance imaging.

\section{Acknowledgements}

This work was financially supported by the National Natural Science Foundation of China $(21275158,21105117)$, the
Innovation Projects of the Chinese Academy of Sciences (KZCX2-EW-206), the 100 Talents Program of the Chinese Academy of Sciences.

\section{Notes and references}

1 Y. Hoshino, T. Kodama, Y. Okahata and J. K. Shea, J. Am. Chem. Soc., 2008, 130, 15242.

2 J. Wang and W. Gao, ACS Nano, 2012, 6, 5745.

3 A. Eldar-Boock, D. Polyak, A. Scomparin and R. SatchiFainaro, Curr. Opin. Biotechnol., 2013, 24, 682.

4 L. Ye and K. Mosbach, Chem. Mater., 2008, 20, 859.

5 L. X. Chen, S. F. Xu and J. H. Li, Chem. Soc. Rev., 2011, 40, 2922. 6 B. Özlem, E. Ünlüera, A. Ersöza, A. Denizlib, R. Demirelc and R. Say, J. Chromatogr. B: Anal. Technol. Biomed. Life Sci., 2013, 934, 46.

7 Z. Zhang, S. F. Xu, J. H. Li, H. Xiong, H. L. Peng and L. X. Chen, J. Agric. Food Chem., 2012, 60, 180.

8 W. J. Cheong, S. H. Yang and F. Ali, J. Sep. Sci., 2013, 36, 609. 9 H. B. Li, Y. L. Li and J. Cheng, Chem. Mater., 2010, 22, 2451. 10 J. H. Li, Z. Zhang, S. F. Xu, L. X. Chen, N. Zhou, H. Xiong and H. L. Peng, J. Mater. Chem., 2011, 21, 19267.

$11 \mathrm{H}$. Henschel, N. Kirsch, J. Hedin-Dahlstrom, M. J. Whitcombe, S. Wikman and A. J. Nicholls, J. Mol. Catal. B: Enzym., 2011, 72, 199.

12 S. J. Li, Y. Ge and A. P. F. Turner, Adv. Funct. Mater., 2011, 21, 1194.

13 B. Sellergren and C. J. Allender, Adv. Drug Delivery Rev., 2005, 57, 1733.

14 M. S. Silva, R. Viveiros, P. I. Morgado, A. Aguiar-Ricardo, I. J. Correia and T. Casimiro, Int. J. Pharm., 2011, 416, 61.

15 M. E. Byrne, J. Z. Hilt and N. A. Peppas, J. Biomed. Mater. Res., Part A, 2008, 84, 137.

16 M. S. Zhang, J. R. Huang, P. Yu and X. Chen, Talanta, 2010, 81, 162.

17 L. Qin, X. W. He, W. Zhang, W. Y. Li and Y. K. Zhang, Anal. Chem., 2009, 81, 7206.

18 X. F. Zhang, X. Z. Du, X. Huang and Z. P. Lv, J. Am. Chem. Soc., 2013, 135, 9248.

19 M. J. Whitcombe, I. Chianella and L. Larcombe, Chem. Soc. Rev., 2011, 40, 1547.

20 E. Verheyen, J. P. Schillemans, M. V. Wijk, M. A. Demeniex, W. E. Hennink and C. F. V. Nostrum, Biomaterials, 2011, 32, 3008.

21 W. Zhang, L. Qin, X. W. He and W. Y. Li, J. Chromatogr. A, 2009, 1216, 4560.

22 K. G. Yang, L. H. Zhang, Z. Liang and Y. K. Zhang, Anal. Bioanal. Chem., 2012, 403, 2173.

23 X. T. Shen, T. C. Zhou and L. Ye, Chem. Commun., 2012, 48, 8198.

24 E. Saridakis and N. E. Chayen, Trends Biotechnol., 2013, 31, 515.

25 X. L. Song, J. H. Li, S. F. Xu, R. J. Ying, J. P. Ma, C. Y. Liao, D. Y. Liu, J. B. Yu and L. X. Chen, Talanta, 2012, 99, 75.

26 H. C. Chen, D. Y. Yuan, Y. Y. Li, M. J. Dong, Z. H. Chai, J. Kong and G. Q. Fu, Anal. Chim. Acta, 2013, 779, 82.

27 J. B. Wu, S. Y. Zang and Y. L. Yi, Sci. Technol., 2013, 66, 434. 
28 X. P. Jia, M. L. Xu, Y. Z. Wang, D. Ran, S. Yang and M. Zhang, Analyst, 2013, 138, 651.

29 L. Qin, X. W. He, W. Zhang, W. Y. Li and Y. K. Zhang, J. Chromatogr. A, 2009, 1216, 807.

30 W. Zhang, X. W. He, W. Y. Li and Y. K. Zhang, Chem. Commun., 2012, 48, 1757.

31 M. H. Lee, J. L. Thomas, M. H. Ho, C. Yuan and H. Y. Lin, ACS Appl. Mater. Interfaces, 2010, 2, 1729.

32 S. F. Xu, J. H. Li, X. L. Song, J. S. Liu, H. Z. Lu and L. X. Chen, Anal. Methods, 2013, 5, 124.

33 S. F. Xu, H. Z. Lu, X. W. Zheng and L. X. Chen, J. Mater. Chem. C, 2013, 1, 4406.

34 X. J. Li, B. L. Zhang, W. Li, X. F. Lei, X. L. Fan, L. Tian, H. P. Zhang and Q. Y. Zhang, Biosens. Bioelectron., 2014, 51, 261.

35 R. MacColl, W. Habig and D. S. Berns, J. Biol. Chem., 1973, 248, 7080.

36 S. Nagaoka, K. Shimizu, H. Kaneko, F. Shibayama, K. Morikawa, Y. Kanamaru, A. Otsuka, T. Hirahashi and T. Kato, J. Nutr., 2005, 135, 2425.
37 N. T. Eriksen, Appl. Microbiol. Biotechnol., 2008, 80, 1.

38 K. S. Lia, Z. Lia, L. Tedescoa and B. Halla, Ecological Informatics, 2013, 15, 22.

39 M. Gantar, D. Simović, S. Djilas, W. W. Gonzalez and J. Miksovska, J. Biotechnol., 2012, 159, 21.

40 H. Deng, X. L. Li, Q. Peng, X. Wang, J. P. Chen and Y. D. Li, Angew. Chem., Int. Ed., 2005, 44, 2782.

41 C. J. Tan, H. G. Chua, K. H. Ker and Y. W. Tong, Anal. Chem., 2008, 80, 683.

42 Y. Q. Wang, L. X. Chen and P. Liu, Chem.-Eur. J., 2012, 18, 5935.

43 M. Danish, R. Hashim, M. N. M. Ibrahim, M. Rafatullah, O. Sulaiman, T. Ahmad, M. Shamsuzzoha and A. Ahmad, J. Chem. Eng. Data, 2011, 56, 3607.

44 X. Q. Cai, J. H. Li, Z. Zhang, F. F. Yang, R. C. Dong and L. X. Chen, ACS Appl. Mater. Interfaces, 2014, 6, 305.

45 X. Q. Cai, J. H. Li, Z. Zhang, G. Wang, X. L. Song, J. M. You and L. X. Chen, Talanta, 2014, 120, 297. 